\title{
Fabry-Pérot resonators for surface plasmon polaritons probed by cathodoluminescence
}

\author{
M. Kuttge, ${ }^{\text {a) }}$ E. J. R. Vesseur, and A. Polman \\ Center for Nanophotonics, FOM-Institute AMOLF, Sciencepark 113, 1098XG Amsterdam, The Netherlands
}

(Received 5 December 2008; accepted 8 April 2009; published online 4 May 2009)

\begin{abstract}
Surface plasmon polariton Fabry-Pérot resonators were made in single-crystalline gold by focused ion beam milling of two parallel $100 \mathrm{~nm}$ deep grooves. The plasmonic cavity modes were spatially and spectrally resolved using cathodoluminescence spectroscopy. Mode numbers up to $n=10$ were observed. The cavity quality factor $Q$ depends strongly on groove depth; the highest $Q=21$ was found for groove depth of $100 \mathrm{~nm}$ at $\lambda=690 \mathrm{~nm}$. The data are consistent with finite-difference time domain calculations that show that the wavelength of maximum reflectivity is strongly correlated with groove depth. () 2009 American Institute of Physics. [DOI: 10.1063/1.3126484]
\end{abstract}

Surface plasmon polaritons (SPPs) are electromagnetic waves bound to the interface between a metal and a dielectric. ${ }^{1}$ The strong coupling between optical radiation and the collective plasmon oscillations of the conduction electrons near the metal surface leads to large field enhancements at the interface. At frequencies close to the plasmon resonance, SPPs possess large wave vectors enabling sub$100 \mathrm{~nm}$ optics at optical frequencies. By varying the metal thickness, the SPP dispersion can be further tailored. SPPs thus enable two-dimensional optics in which optical information can be guided and processed at the nanoscale. While the propagation of SPPs has been well studied, ${ }^{2-5}$ a next challenge is to obtain control over the confinement of SPPs.

So far, reflectors composed of arrays of nanoparticles ${ }^{6}$ and Bragg cavities ${ }^{7}$ composed of arrays of very shallow grooves or ridges have been studied to achieve SPP confinement. Weeber et $_{\text {al. }}{ }^{7}$ showed that two parallel ridge gratings can act as Bragg-mirrors and can confine plasmons between the mirrors. Because of the narrowband reflection of these gratings the field enhancement was only observed for a small wavelength range.

In this letter, we use a single deep groove in the surface of single crystalline gold as an effective mirror for surface plasmons. By placing two parallel grooves on the surface we construct a Fabry-Pérot resonator for SPPs. We use cathodoluminescence (CL) imaging spectroscopy ${ }^{3,4}$ to excite the resonators and determine the spatially resolved cavity field profile. From the observed field profile we determine the mode numbers and cavity quality factor. Studies of the quality factor as a function of groove depth show a maximum of $Q=15$ at a groove depth of $100 \mathrm{~nm}$. Finite-difference time domain (FDTD) calculations of the groove reflectivity show an increase of reflectivity for these depths, supporting the experimental observations.

Experiments were performed on a single-crystal Au pellet of $1 \mathrm{~mm}$ thickness (effectively semi-infinite for optical fields) of which the surface was chemically polished down to nanometer roughness. Two parallel linear grooves were milled into the surface with a $30 \mathrm{keV} \mathrm{Ga}$ focused ion beam [see schematic in Fig. 1(a)]. The groove separation $w$ was $3000 \mathrm{~nm}$, the depth $d$ was $100 \mathrm{~nm}$, and the groove width was $50 \mathrm{~nm}$ full width at half maximum (FWHM). The groove

${ }^{a)}$ Electronic mail: kuttge@amolf.nl. shape and geometry were determined from scanning electron microscopy (SEM) images of a cross section of a groove pair [see Fig. 1(b)]. To fabricate the cross section a box was milled into the surface next to the grooves. To achieve the proper contrast to image the gold profile, platinum was first deposited over the grooves with focused-ion beam assisted deposition.

Spatially resolved CL spectroscopy was performed in a SEM using a $30 \mathrm{keV}$ electron beam from a field-emission source focused onto the sample to a $\sim 5 \mathrm{~nm}$ diameter spot. The electron beam effectively acts as a point source for SPPs with a broadband spectrum ranging from the SPP resonance at $550 \mathrm{~nm}$ well into the infrared. ${ }^{3,4}$ The scanning electron beam passes through a hole in a parabolic mirror that is positioned above the sample. The mirror collects light emitted from the sample, that is then focused on the entrance slit of a monochromator and spectrally resolved using a charge coupled device array detector (bandwidth $\approx 10 \mathrm{~nm}$ ). The mirror acceptance solid angle is $1.42 \pi$. The measured CL spectra were corrected for system response, which was determined by normalizing the measured raw data from a planar $\mathrm{Au}$ sample (no grooves) to the calculated transition radiation spectrum for $30 \mathrm{keV}$ electron-irradiated Au. ${ }^{8}$ The experimental count rate was $100-500$ counts/s per wave-

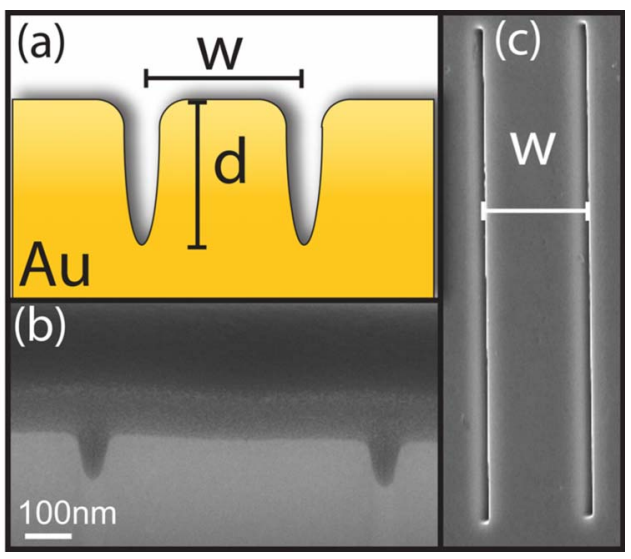

FIG. 1. (Color online) (a) Schematic of the double-groove Fabry-Pérot cavity in a gold surface. (b) Cross-section SEM image of the double groove structure. The grooves were filled with platinum and then a box was structured to allow side view. (c) SEM top-view of the double groove structure. $w$ indicates the center-to-center groove spacing. 


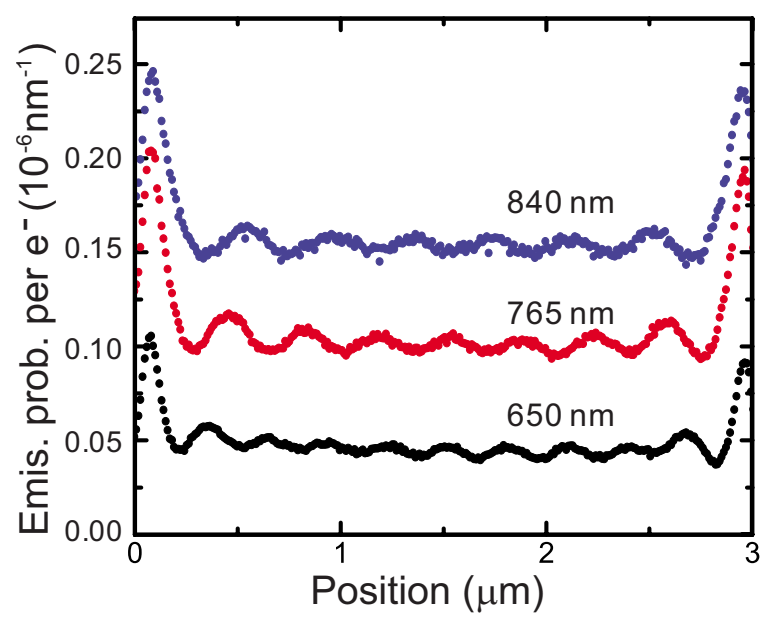

FIG. 2. (Color online) CL line scans (30 keV electron beam) over a double groove structure $(w=3000 \mathrm{~nm}$ and $d=100 \mathrm{~nm})$ for wavelengths of $650 \mathrm{~nm}$ (black symbols), $765 \mathrm{~nm}$ (red symbols), and $840 \mathrm{~nm}$ (blue symbols). The grooves are located at 0 and $3 \mu \mathrm{m}$. The curves are shifted by $0.05(765 \mathrm{~nm})$ and $1.0(840 \mathrm{~nm})$ for clarity.

length channel at an electron beam current of $28 \mathrm{nA}$.

We measured the CL spectra as a function of electron beam position along line scans perpendicular to the groove pairs with a step size of $5 \mathrm{~nm}$ and integration time of $1 \mathrm{~s}$. Figure 2 shows the CL intensity for line scans over the double grooves for three different wavelengths (650, 765, and $840 \mathrm{~nm}$ ). The peaks in the CL intensity coincide with the position of the grooves. Between the grooves a periodic pattern of the CL emission is observed for all wavelengths. The period of the oscillation equals approximately half the freespace wavelength for all three studied wavelengths.

The oscillations in the CL intensity are consistent with a standing SPP wave between the two grooves of the structure. The double-groove structure thus acts as a Fabry-Pérot resonator with the grooves acting as SPP reflectors. The interference condition for the SPP Fabry-Pérot cavity is given by $2 d k_{\mathrm{SPP}}+2 \phi=2 \pi n$ with $d$ the cavity length, $k_{\mathrm{SPP}}$ is the SPP wave vector, $\phi$ is a phase shift upon reflection, and $n$ is the mode number. Note that in CL spectroscopy the spatial resolution results from the known profile of the exciting electron beam. The oscillations in the radiation that are observed in the far field are due to the fact that the electron preferentially excites SPPs at positions of maximal electric field amplitude, i.e., the nodes in the standing wave pattern.

Figure 2 also shows that the visibility of the interference fringes increases with increasing wavelength. This is consistent with the larger SPP propagation length for larger wavelengths. For the shortest wavelengths the amplitude of the oscillations increases closer to the grooves again consistent with the shorter propagation length. Indeed, the SPP propagation length at $650 \mathrm{~nm}$ is a factor of 5 shorter than at $840 \mathrm{~nm}^{5}$

To further study the Fabry-Pérot interference condition, we structured groove pairs with different groove separations into the gold surface, keeping the groove depth and width constant. Figure 3 shows the measured CL intensity as a function of position and wavelength for three groove separations of 1000, 2000, and $3000 \mathrm{~nm}$, respectively. As can be seen, for wavelengths above $600 \mathrm{~nm}$ the measurements clearly show the standing wave pattern in the CL intensity. In the region below $600 \mathrm{~nm}$ the CL intensity decreases as a

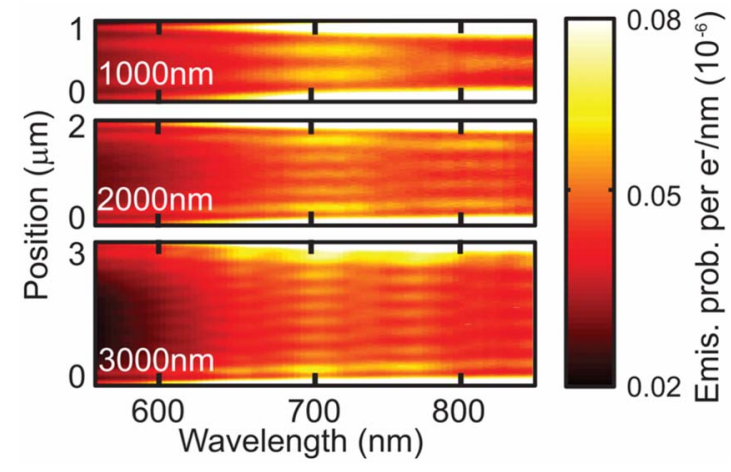

FIG. 3. (Color online) CL as a function of position and wavelength for line scans across three SPP Fabry-Pérot resonators with different center-tocenter groove separation $(w=1000,2000$, and $3000 \mathrm{~nm})$. Multimode spectra with $n=2-10$ are observed.

function of distance to the grooves showing only weak oscillations in particular for the largest cavity. In this region the SPP propagation length is shorter than the cavity length and no standing waves can form.

From the intensity maxima observed in Fig. 3 the resonant modes can be identified. For example, for the $1000 \mathrm{~nm}$ wide resonator two maxima are observed in the standing wave pattern at $700 \mathrm{~nm}$, corresponding to a $n=3$ mode. Similarly, $n=6$ and $n=9$ modes are observed at $700 \mathrm{~nm}$ for the 2000 and $3000 \mathrm{~nm}$ wide resonators. Due to the smaller free spectral range for the $3000 \mathrm{~nm}$ wide cavity a broad distribution of modes is observed around $700 \mathrm{~nm}$ ranging from $n=7-11$.

It is interesting to note that a $n=3$ mode at $700 \mathrm{~nm}$ would correspond to a resonator width of $1020 \mathrm{~nm}$ (taking into account the dispersion of SPPs at $700 \mathrm{~nm}$ ) which is 20 $\mathrm{nm}$ wider than the center-to-center spacing of the grooves. This implies that the effective cavity length is larger than the center-to-center spacing. This is consistent with the fact that the SPPs "probe" the depth of the grooves in their reflection. In this model the effective cavity length offset should be identical $(20 \mathrm{~nm})$ for all cavity lengths. Indeed, the $n=6$ and $n=9$ modes shift to slightly shorter wavelengths for the 2000 and $3000 \mathrm{~nm}$ resonators.

Next, we determined the quality factor of the resonators. To do so, we have structured a range of resonators into the single-crystal gold surface for which we varied the groove depth and thereby the reflectivity. The depth was varied in the range of 20-200 nm keeping the width approximately constant to $50 \mathrm{~nm}$ FWHM. The CL intensity was measured on a line perpendicular to the grooves with a step size of 10 $\mathrm{nm}$. The CL line scans show interference fringes as in Fig. 2 for all wavelengths but with different degrees of visibility. The visibility increases with increasing depth for groove depths up to $100-120 \mathrm{~nm}$. For deeper grooves the visibility decreases.

To determine the quality factors we performed a factor analysis ${ }^{9}$ on the spectral CL line scan for a groove depth of $100 \mathrm{~nm}$ and width of $3000 \mathrm{~nm}$ (see Fig. 3) to determine the resonance spectra. Five significant resonances at wavelengths of $640 \mathrm{~nm}(n=10), 690 \mathrm{~nm}(n=9), 765 \mathrm{~nm}(n=8)$, $870 \mathrm{~nm}(n=7)$, and $980 \mathrm{~nm}(n=6)$ were found to represent the data well. We reconstructed the experimental data from the resonances by fitting their line width and spatial position. The quality factor for each resonance is then given by the 


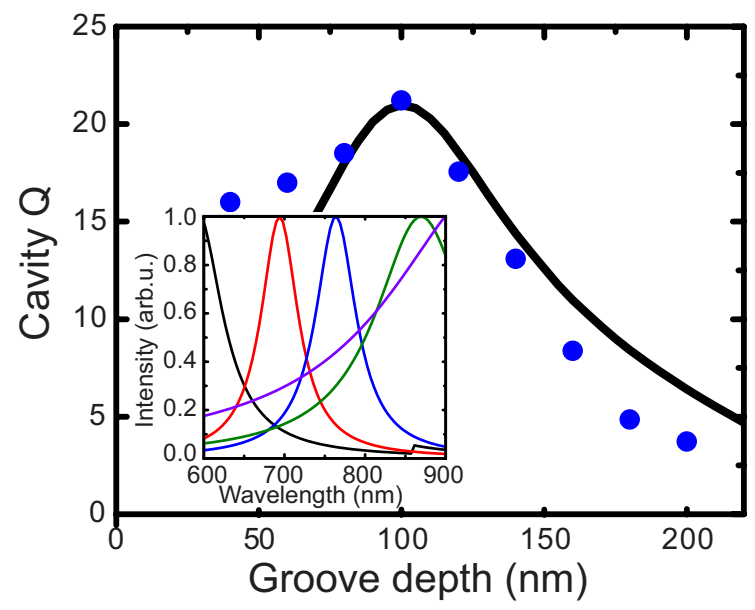

FIG. 4. (Color online) Quality factor for groove resonators for the resonance at $690 \mathrm{~nm}$ as a function of groove depth, extracted from CL line scans (symbols). The black line shows the quality factor calculated from the theoretical groove reflectivity and cavity losses. The inset shows the resonance spectra at 640,690,765, 870, and $980 \mathrm{~nm}$ derived from CL data for a resonator with $100 \mathrm{~nm}$ deep grooves $(w=3000 \mathrm{~nm}$, last panel in Fig. 3).

resonance wavelength divided by the line width. The inset of Fig. 4 shows the five resonance spectra. The smallest line widths are observed for the central modes at 690 and 765 $\mathrm{nm}$. Lower quality factors are observed for the 640 as well as the 870 and $890 \mathrm{~nm}$ modes.

The quality factor of the resonator is determined by propagation losses and the reflectivity of the mirrors. For the shortest wavelength $(640 \mathrm{~nm})$, propagation losses dominate, as was also apparent from Fig. 2 and a larger line width is observed (see inset of Fig. 4). To determine the reflectivity of a single groove for SPPs we have performed FDTD calculations. We used a two-dimensional simulation with the groove profile modeled according to the SEM images of the cross cuts. The SPP mode was generated at a distance of $2 \mu \mathrm{m}$ away from the groove. Reflection and transmission of the groove were monitored using field monitors past the groove and behind the source. Figure 5 shows the calculated groove reflectivity as a function of wavelength and groove depth. For each wavelength a maximum of the reflection coefficient is observed for a certain groove depth. The wavelength of the maximum increases with groove depth pointing to a reso-

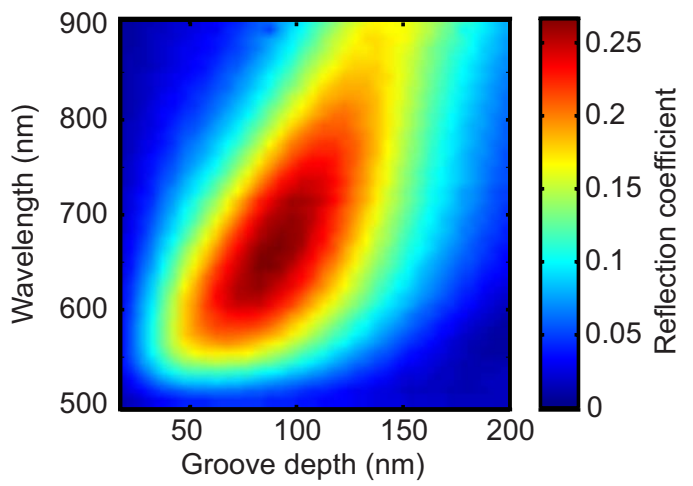

FIG. 5. (Color online) Reflection coefficient for SPPs at a single $100 \mathrm{~nm}$ wide groove in gold as a function of groove depth and SPP wavelength, as determined from FDTD calculations. nance condition for the reflectivity maximum. Indeed, further calculations showed that the reflectivity is related to resonant localized modes inside the groove cavities. ${ }^{10}$ The wavelength of the maximum increases with groove depth. The maximum reflection coefficient for $100 \mathrm{~nm}$ deep grooves is observed in the range of $650-700 \mathrm{~nm}$. This is consistent with the smaller line width observed for the modes in that wavelength range in the inset of Fig. 4 or, correspondingly, the fact that the highest visibility resonances in Fig. 3 are observed around $700 \mathrm{~nm}$. For wavelengths longer than $800 \mathrm{~nm}$ the reflectivity decreases as the incoming SPP is only slightly perturbed by the groove.

We have performed the factor analysis for the line scans of the other depths (20-200 nm). Figure 4 shows the quality factor for the resonance near $690 \mathrm{~nm}$ as a function of groove depth. The quality factor increases for increasing groove depth reaching a maximum of $Q=21$ at $100 \mathrm{~nm}$ and decreases for larger groove depths. This is again consistent with Fig. 5.

Taking into account the reflectivity of the grooves and damping by the metal the theoretical quality factor can be calculated. In Fig. 4 we present the theoretical quality factor that shows a maximum of $Q=21$ for $100 \mathrm{~nm}$ deep grooves in good agreements with experiments.

In conclusion, we have investigated Fabry-Pérot resonators for SPPs consisting of two parallel grooves. CL allowed direct imaging of the field profile inside the cavities and determine mode numbers up to $n=10$. The cavity quality factor was found to depend strongly on groove depth and wavelength, showing a maximum of $Q=21$ at $700 \mathrm{~nm}$ for a groove depth of 100-120 nm. Further work will focus on achieving understanding of the details of the groove reflection of SPPs, taking into account localized modes inside the groove cavities. ${ }^{10}$

Harry Atwater and Henry Lezec are acknowledged for support in the previous stages of sample fabrication. This work is part of the research program of FOM, which is financially supported by NWO. This work is also supported by NANONED, a nanotechnology program funded by the Dutch Ministry of Economic Affairs, and the MMN research program which is partially financed by FEI Co.

${ }^{1}$ H. Raether, Surface Plasmons on Smooth and Rough Surfaces and on Gratings (Springer, New York, 1988)

${ }^{2}$ J. R. Krenn, H. Ditlbacher, G. Schider, A. Hohenau, A. Leitner, and F. R. Aussenegg, J. Microsc. 209, 167 (2003).

${ }^{3}$ J. T. van Wijngaarden, E. Verhagen, A. Polman, C. E. Ross, H. J. Lezec, and H. A. Atwater, Appl. Phys. Lett. 88, 221111 (2006).

${ }^{4}$ M. V. Bashevoy, F. Jonsson, A. V. Krasavin, N. I. Zheludev, Y. Chen, and M. I. Stockman, Nano Lett. 6, 1113 (2006).

${ }^{5}$ M. Kuttge, E. J. R. Vesseur, J. Verhoeven, H. J. Lezec, H. A. Atwater, and A. Polman, Appl. Phys. Lett. 93, 113110 (2008).

${ }^{6}$ A. Drezet, A. L. Stepanov, H. Ditlbacher, A. Hohenau, B. Steinberger, F. R. Aussenegg, A. Leitner, and J. R. Krenn, Appl. Phys. Lett. 86, 074104 (2005).

${ }^{7}$ J.-C. Weeber, A. Bouhelier, G. Colas des Francs, L. Markey, and A. Dereux, Nano Lett. 7, 1352 (2007).

${ }^{8}$ N. Yamamoto, H. Sugiyama, and A. Toda, Proc. R. Soc. London, Ser. A 452, 2279 (1996)

${ }^{9}$ E. R. Malinowski, Factor Analysis in Chemistry (Wiley, New York, 1991).

${ }^{10}$ M. Kuttge, F. J. García de Abajo, and A. Polman, "How grooves reflect and confine surface plasmon polaritons," Opt. Express (submitted). 